\title{
Rehabilitación psicosocial. Perspectiva actual y directrices en la Reforma Psiquiátrica
}

\author{
Mariano Hernández Monsalve \\ Comunidad de Madrid - Servicio Madrileno de Salud \\ Servicio de Salud Mental de Tetuán
}

\begin{abstract}
Resumen
Se describen las etapas que ido atravesando la rehabilitación psicosocial en España, con especial atención a su paralelismo con la transformación de los servicios públicos de salud que se inició en 1986 con la promulgación de la Ley General de Sanidad (LGS) en 1986 y, especialmente, con el desarrollo de la Reforma Psiquiátrica que se inició formalmente con el Informe Ministerial para la Reforma Psiquiátrica (1985) y con la citada LGS. Se revisan los aspectos más relevantes del proceso de despliegue de los nuevos servicios de rehabilitación, y el nuevo escenario asistencial tras la publicación de la Estrategia en Salud Mental del Sistema Nacional de Salud (2007), incluyendo la rehabilitación laboral de personas con enfermedad mental, finalizando con una estimación de las perspectivas de futuro.
\end{abstract}

Palabras claves: rehabilitación psicosocial, estrategia en salud mental, sistema nacional de salud.

\begin{abstract}
Psychosocial rehabilitation. Current perspectives and guidelines in the Psychiatric Reform.

It describes the stages gone through psychosocial rehabilitation in Spain with special attention to parallels with the transformation of public health services that began in 1986 with the enactment of the General Health Law in 1986 and especially with the development of the Psychiatric Reform which began formally with the Ministerial Report for Psychiatric Reform (1985) and with the above GHL. We review the relevant aspects of the deployment process of new rehabilitation services, and the new stage of care following the publication of the Mental Health Strategy in the National Health System (2007), including the vocational rehabilitation of people with mental illness ending with an estimate of future prospects.
\end{abstract}

Keywords: psychosocial rehabilitation, mental health strategy, national health system

$\mathrm{L}$ a rehabilitación psicosocial (RPS) ha demostrado ser, en la historia reciente de la psiquiatría y la salud mental, un ingrediente clave en la eficacia de los procesos de desinstitucionalización, recuperación e inclusión social de las personas afectas por trastorno mental grave y persistente (en adelante TMG). La RPS es una dimensión de la intervención en salud mental; se refiere a un conjunto de estrategias para afrontar las dificultades para el desempeño de la vida diaria (para la tarea del vivir) de las personas que padecen problemas persistentes de salud mental, de suficiente gravedad como para limitar el desarrollo de su vida diaria y de sus objetivos personales, y que pone en práctica muy diversas técnicas para ello (desde técnicas específicas de desarrollo de habilidades sociales o de estimulación cognitiva, o de psicoeducación familiar, por ejemplo, hasta otras menos específicas y/o de amplio espectro como el acompañamiento terapéutico, o el seguimiento comunitario), además de realizarse desde unos valores como son los clásicos principios de autonomía, normalización, responsabilización; o las más recientemente reconocidos como los derivados de la perspectiva de la recuperación.
Aún cuando la RPS se identifica y define como tal en época reciente, hacia mediados del siglo pasado, podemos reconocer antecedentes históricos remotos de lo que hoy conocemos por RPS en épocas de institucionalización psiquiátrica, en la terapia moral, en las prácticas de "terapia ocupacional" y en la llamada "laborterapia" por cuanto representaron intentos de activar ciertas potencialidades de los pacientes; en épocas más recientes, se fueron definiendo otras perspectivas más acordes con las necesidades de inserción social y laboral de los pacientes, de modo que se fue hablando de rehabilitación industrial o de rehabilitación vocacional.

\section{Algunos aspectos conceptuales acerca de la Rehabilitación Psicosocial}

Desde su comienzo la rehabilitación ha ofrecido perfiles a menudo controvertidos, referidos a su propio núcleo, a los escenarios y las actores de la rehabilitación, lo que explica algunas dificultades en el despliegue de programas y servicios A modo de síntesis, estos son alguno de los aspectos más controvertidos: 
La separación de tratamiento y rehabilitación que a menudo se refleja en distancia en la organización de servicios (que pueden incluso constituir dos redes paralelas) y en la financiación. Entre las razones que han empujado a esta separación, las dos principales en mi opinión son: por una parte, los hallazgos clínicos que mostraban una evolución "disociada" de síntomas y nivel de adaptación, y por otra parte, la doble consideración de la persona que padece el problema de salud mental, como enfermo (por presentar síntomas) y como discapacitado, con la consiguiente dificultad para los responsables de abordar cada uno de los problemas (el primero - la enfermedad, los síntomas desde los servicios de salud; el segundo - la discapacidad - desde los servicios sociales). La forma de abordar esta disyuntiva es una de las piedras de toque de la eficacia y de la calidad de los servicios (pues obviamente, la persona y su contexto son los mismos en ambos casos). Cada vez más voces claman a favor de trabajar en pro de un abordaje conjunto de ambas dimensiones (Esteban, 2002; Harding \& McCrory, 2008; Shephred, 1996; Uriarte, 2007).

Otra cuestión importante es la importancia relativa, en todo proyecto de rehabilitación y reinserción, de la intervención profesional y las redes sociales naturales (Saraceno \& Montero, 1993). El clásico principio de no generar espacios ni dispositivos específicos hasta no haber agotado las posibilidades que ofrece el entorno normalizado no siempre se tiene en cuenta en la práctica.

En lo que a la implantación y práctica de la RPS en España se refiere, podemos reconocer tres etapas:

La etapa pre-reforma. Década de los setenta y ochenta hasta la publicación del Informe Ministerial de la Reforma psiquiátrica, en 1985.

El comienzo de la reforma "oficial”. Desde la Ley General de Sanidad (LGS), 1986, hasta la aprobación, por el Consejo Interterritorial $^{1}$, del documento Estrategia en Salud Mental del Sistema Nacional de Salud, en diciembre del 2007.

Desde la publicación del documento de la Estrategia hasta la actualidad.

\section{La pre-reforma psiquiátrica}

Consideramos esa etapa de forma un tanto imprecisa (década de los setenta y principios de los ochenta), a los últimos tiempos de psiquiatría institucional en España, cuando la atención psiquiátrica estaba prácticamente reducida a los hospitales psiquiátricos y estaba regentado por la "beneficiencia"2 o por entidades privadas, fuera del sistema público de salud (en aquellos años, financiado y gestionado por el "Seguro Obligatorio de Enfermedad), excepto a través de las consultas de Neuropsiquiatría, que atendían indistintamente, en consultas de unos minutos - de dos horas diarias, a pacientes que acudían con problemas de cualquiera de las dos especialidades. Esta época coincide a su vez con los últimos años del franquismo y el comienzo del cambio político conocido como la transición. Se dieron sucesivos intentos de cambio, como expresión de la voluntad de modernizar, humanizar, democratizar la atención psiquiátrica, si bien esos intentos no cuajaron hasta años después. Las experiencias más significativas de esos años están accesibles en diversas publicaciones de la Asociación Española de Neuropsiquiatría (Espinosa, 1986; González de Chávez, 1980) y otras (Desviat, 1994; García, Espino, \& Lara, 1998; Rebolledo, 1997, Rodríguez, 1997).

El punto de inflexión "formal" hacia el nuevo modelo de salud mental comunitaria, y su inclusión en el sistema público de salud se da con la publicación del "Informe de la Comisión Ministerial para la Reforma Psiquiátrica” (1985 y la posterior promulgación de la Ley General de Sanidad en 1986). En lo que se refiere a la rehabilitación, el citado informe está teñido de los valores de la desinstitucionalización, pero no considera la RPS en el mismo rango en que hoy lo hacemos, pues mantiene una visión en la que predomina la rehabilitación como actividad a desarrollar en los hospitales - para preparar la salida de los pacientes - aunque también incluye referencias a potenciar al máximo la atención en el medio comunitario.

\section{Segunda etapa: llega la Ley General de Sanidad (LGS) y comienza "oficialmente" la reforma psiquiátrica en el conjunto del Estado.}

Las reformas siguen avanzando, pero en lo que a la RPS se refiere el progreso es dubitativo, irregular, a trompicones. Su evolución es paralela a la transformación de un Estado central a un Estado autonómico (17 Comunidades Autónomas más las ciudades africanas de Ceuta y Melilla), con progresiva descentralización. Los recursos de rehabilitación caen a menudo en las fisuras entre los servicios sanitarios y los servicios sociales, tanto desde el punto de vista de la planificación como de la gestión de la financiación. La mencionada LGS, incluye en el Art 20 las siguientes referencias a la rehabilitación:

Se desarrollarán los servicios de rehabilitación y reinserción social necesarios para una adecuada atención integral a los problemas del enfermo mental, buscando la necesaria coordinación con los servicios sociales. Los servicios de salud mental y de atención psiquiátrica del sistema sanitario general cubrirán, asimismo, en coordinación con los servicios sociales, los aspectos de prevención primaria y de atención a los problemas psicosociales que acompañan a la pérdida de la salud en general (Ley General de Sanidad, 1986, artículo 20, apartados 3 y 4; BOE 102, p. 15211).

Sin embargo, este marco legal no supuso la puesta en marcha inmediata de dispositivos de rehabilitación: la primer etapa de la reforma oficial significó sobre todo un despliegue de nuevos servicios de salud mental comunitarios y unidades de hospitalización psiquiátrica en los hospitales generales, siendo muy escaso el desarrollo de dispositivos intermedios - de rehabilitación y de apoyo social a la reinserción.

Buena expresión de la insuficiencia de la rehabilitación en esos años es la crítica del Defensor del Pueblo del año 1991 y los comentarios de uno de los líderes del movimiento de familiares, en el año 2000, así como en el análisis de situación y reconocimiento de puntos críticos que, formalmente, se reconoce en el documento de la Estrategia en Salud Mental del Sistema Nacional de Salud $(2007)^{3}$, al que luego me referiré con más detalle. A finales de 1991 el Defensor del 
Pueblo Español, en su Informe sobre la situación jurídica $y$ asistencial del enfermo mental en España, señalaba la carencia de dispositivos intermedios, la escasa disponibilidad de estructuras de carácter social que estaba condicionando los planes de desinstitucionalización de los pacientes internados y la persistencia de hospitales psiquiátricos que apenas contaban con algún psiquiatra en su plantilla, y en condiciones de habitabilidad bajo mínimos (Defensor del Pueblo, 1992). Otros informes tales como del Defensor del Pueblo Andaluz, o el del Ararteko en el País Vasco, apuntaron en la misma dirección. Las reformas avanzaban más a nivel declarativo que en los hechos. Por su parte, Avelino Fernández, que había sido hasta poco tiempo antes presidente de $\mathrm{AMAFE}^{4}$, escribía aún en el 2000:

la desinstitucionalización y con ella el sistema de psiquiatría comunitaria a que da paso, se convierte, por sus enormes defectos de aplicación, y pese al excelente espíritu que la promovió, en una auténtica pesadilla para las familias implicadas, que a menudo nos preguntamos ¿ qué está más lejos de la realidad, la mente del enfermo o el sistema montado para atenderle? ....en un simple traslado del crónico desde la institución al hogar (Fernández, 2000, p. 335).

A lo largo de la década de los 1990 y primeros años del siglo XXI asistimos a amplia producción teórica, especialmente por parte de la Asociación Española de Neuropsiquiatría (AEN) y de la recién nacida Federación Española de Asociaciones de Rehabilitación Psicosocial (FEARP), siendo en mi opinión el documento más significativo el "Cuaderno Técnico sobre RPS” publicado por la AEN en 2002 (Gisbert, 2002), en cuya redacción participaron profesionales de ambas asociaciones, que viene a representar la expresión de una perspectiva sobre RPS que tenia en aquellos momentos el mayor consenso entre los distintos grupos que estaban implicados en ello en el conjunto del Estado Español, más allá de las diferencias existentes entre las Comunidades Autónomas. En este documento se define el objetivo como el de "mejorar la calidad de vida de estas personas con enfermedad mental grave y persistente, y ayudarlas a asumir la responsabilidad de sus propias vidas y a actuar en la comunidad tan activamente como sea posible y a manejarse con la mayor independencia posible en su entorno social" (Gisbert, 2002, p.21). Desde esta perspectiva, los programas de rehabilitación psicosocial están concebidos para reforzar las habilidades del individuo y para desarrollar los apoyos ambientales necesarios para mantenerse en su entorno.

Ese documento incluye una propuesta de estrategia de desarrollo de servicios, de modo que configuren una red que incluya programas de RPS en los centros de salud mental comunitarios y una variada red de dispositivos específicos. Y concluye con una serie de recomendaciones, entre las que me parecen importante destacar: el que las administraciones lo aborden como una prioridad política, así como la urgencia en apoyar el movimiento asociativo y proceder a la formación de los profesionales en este ámbito de intervención.

A lo largo de esta segunda etapa (1986-2006) el panorama en el territorio español es muy heterogéneo. Pero en varios lugares se lograron concretar experiencias exitosas que marcan los principales hitos de esta etapa.
La reforma psiquiátrica en Leganés (Madrid). Se propuso el cierre de un hospital psiquiátrico y la creación de una red de servicios de salud mental comunitarios, que incluyera un potente conjunto de programas y servicios de RPS. Los rasgos principales de la estrategia de reforma seguida allí son los siguientes:

1. Los programas de rehabilitación fueron desde el principio una de las prioridades de la reforma de Leganés, por considerar que de sus resultados dependería en gran parte la credibilidad y viabilidad del proyecto de servicios de salud mental alternativos (ii no complementarios!!) al hospital psiquiátrico.

2. Los programas y dispositivos de RPS y apoyo comunitario se desarrollaron desde los mismos presupuestos y estructura organizativa que el resto de programas de salud mental comunitaria del área, bajo la misma dirección, compartiendo las mismas comisiones de trabajo así como los programas de coordinación intra e interinstitucional, así como los de formación (de residentes de psiquiatría y de psicología clínica, de enfermería de salud mental) y de investigación epidemiológica y clínica.

3. Se estableció una fluida continuidad entre los programas de RPS a cargo de los equipos de salud mental comunitaria (los programas de seguimiento), los programas de RPS especializados de los dispositivos específicos (en centro de día, unidad hospitalaria de rehabilitación), los de apoyo comunitario (pisos supervisados, residencia asistida) y con las actividades de "gestión ciudadana" tales como el grupo de autoayuda de los familiares o el club social de los pacientes. Se iniciaron también actividades empresariales para la inserción laboral, dentro del programa europeo Horizon, que no pudieron continuarse al finalizar el proyecto europeo por falta de apoyo de la Administración (de la comunidad autónoma de Madrid). Se priorizó el mantener una perspectiva y visión de conjunto del trabajo en red, propiciando al máximo la continuidad de cuidados y las intervenciones en contextos normalizados, en concordancia con los servicios sociales y otras instancias de implantación local. El impacto de ese proceso en ámbito local e internacional son bien conocidos (Desviat, 1994).

La experiencia del Barrio de Gracia en Barcelona. desplegada por la asociación privada, de ámbito social, ARAPDIS (Blasi Ras, 2000), que suponía una isla de rehabilitación psicosocial comunitaria en la Cataluña de los años ochenta, que pudo demostrar que la inserción social, laboral y residencial de los pacientes mentales era posible, si bien la debilidad de aquél proyecto residía precisamente en su carácter de "excepción" en el conjunto de una red de servicios de salud mental, que en aquellos años estaba escasamente articulada en Cataluña.

La experiencia andaluza. la primera comunidad autónoma española que cierra todos los hospitales psiquiátricos públicos y crea, a instancias del Parlamento Andaluz, en 1993, una fundación, La Fundación Andaluza para la Integración Social del Enfermo Mental (FAISEM), como institución pública de la Junta de Andalucía (el Gobierno andaluz) vinculada a las Consejerías de Salud, Asuntos Sociales, Empleo y Desarrollo Tecnológico, y Economía y Hacienda para ocuparse de la inserción ocupacional, laboral (creación de empresas sociales, 
y desarrollo de cursos de formación profesional) y residencial ( pisos, casa-hogar, residencias), junto a otros programas de fomento de las relaciones sociales (club sociales, actividades de ocio). Años más tarde, se crearía en Castilla La Mancha una Fundación análoga (FISLEM) ${ }^{5}$.

\section{La rehabilitación en la "Estrategia en Salud Mental del SNS” (2006 hasta la actualidad)}

La Estrategia en Salud Mental surge como necesidad de actualizar la situación asistencial en los servicios públicos de salud mental y de rehabilitación psicosocial, en el conjunto del Estado, consecuencia de una decisión política que tiene en cuenta el que, tras el informe de la Comisión Ministerial, ya aludido y la Ley General de Sanidad ha habido importantes avances y logros , a la vez que importantes desigualdades en aspectos claves de la reforma siendo uno de ellos el que se refiere a la situación en que se encuentran los afectados por trastornos más graves y más crónicos y las poblaciones socialmente más precarias. La Estrategia se propone también armonizar la situación asistencial entre las diversas Comunidades Autónomas, buscando los elementos comunes e intentado garantizar parámetros de equidad y calidad. Es un momento de nuevo gobierno socialista (se inició en 2004) coincidiendo con otras "estrategias" (cardiopatía isquémica, cáncer). Y coincidiendo además con novedades en la perspectiva europea (La Conferencia Europea - OMS Europa - y Plan de Acción de Helsinki en enero de 2005 (World Health Organization, 2005); Libro Verde de la Salud Mental - documento de trabajo de la Comisión Europea - en 2006 (European Communities, Health and Consumer Protection Directorate-General, 2006) y Pacto Europeo por la Salud Mental - propiciado por la Comisión Europea, en junio de 2008 (European Comission, 2008).

El objetivo integrador del proyecto se plasmó desde el principio en la propia constitución del grupo que habría de redactarlo, al constituir, junto a un Comité Técnico de Redacción, formado por representantes de asociaciones de profesionales y de familiares y personas con enfermedad mental y expertos, otro Comité Institucional constituido por los representantes de todas las Comunidades Autónomas. En cuanto a su análisis de partida, entres los aspectos más significativos encontramos: el reconocimiento de los cambios y de los límites de las intervenciones profesionales centradas en el tratamiento y la rehabilitación en sus versiones más estrictamente "técnicas", proponiendo aceptar la importancia de poner la vista en el paradigma de la recuperación, y conectar con la perspectiva de promoción y prevención. Y haciendo a su vez una declaración de principios y valores: autonomía, continuidad, accesibilidad, comprensividad, equidad, recuperación personal, responsabilización y calidad. Así, se afirma en el informe que

con el cierre de las grandes instituciones, la vida de las personas con enfermedades mentales graves, que otrora se habría desenvuelto entre muros, ahora se desenvuelve en sus hogares familiares o en residencias y, con ello, sus necesidades se han ido acercando progresivamente a la normalidad, esto es, ajustándose al conjunto de necesidades de la población sana: necesidad de recibir una educación, de trabajar, de poder ganarse la vida, de relacionarse, de ocupar el tiempo libre y, por supuesto, de recibir tratamiento adecuado, incluyendo el rehabilitador. Con todo, las necesidades de estas personas continúan siendo pensadas por los y las profesionales, identificadas por instrumentos desarrollados por ellos y ellas, y satisfechas mediante prestaciones, programas y servicios sanitarios y sociales ad hoc", y reconociendo también la" creciente conciencia de ciudadanía y de sensibilidad hacia el respeto de los derechos humanos, liderado por las asociaciones de los consumidores de los servicios sanitarios. El núcleo de este movimiento social reside en el papel protagonista que los usuarios y sus familiares juegan en relación con la definición de sus necesidades, y la planificación, creación y gestión de servicios para satisfacerlas (Ministerio de Sanidad y Consumo, 2007, p. 27).

Entre las asignaturas pendientes, el documento de la Estrategia reconoce que "el desarrollo de recursos alternativos al hospital psiquiátrico dentro del tejido comunitario se está realizando con lentitud, con importantes diferencias entre Comunidades Autónomas y, en algunos casos, sin responder a criterios de planificación; y el insuficiente desarrollo comunitario, expresado en la poca participación real de los usuarios y usuarias (y sus organizaciones) y de nivel local (Ayuntamientos)".

Otro aspecto importante es el reconocimiento de la carga que supone la enfermedad para las familias, carga objetiva (la económica, y la repercusión en menor tiempo para formación, trabajo y ocio de otros miembros de la familia) y carga subjetiva, de sufrimiento y "duelo", presentando esta población (de cuidadores) una morbilidad psiquiátrica doble que la esperada en población general con parámetros sociodemográficos similares. En lo que respecta a la carga económica, aun con las dificultades de la estimación precisa de la misma, la información disponible dice que la persona con esquizofrenia genera mayor carga económica a la familia que a la Administración. Y contempla también la necesidad de considerar nuevos derechos que se han ido reconociendo en los últimos años, como es el caso del derecho al "consentimiento informado", en pie de igualdad que el resto de otros usuarios-pacientes del sistema sanitario.

En España la atención proporcionada por cuidadores informales es el 88\%. El cuidador suele ser mujer entre 50-65 años (la edad de las madres y padres cuidadores está aumentando, con las dificultades que cabe esperar) con nivel cultural medio; y se estima que dedica a esta tarea más de 60 horas semanales durante años. Y otro factor añadido muy importante es que en una proporción importante de estas familias en el 37\%), la fuente principal de ingresos económicos son las "pensiones no contributivas" (es decir, no derivadas de condición de haber sido trabajador, que son de escasa cuantía), por lo que difícilmente pueden buscar ayuda por sus propios medios. La Estrategia identifica también una serie de "puntos críticos", que respecto de lo que ahora nos atañe se resumen en los siguientes:

a) Falta de recursos comunitarios supone sobrecarga de las familias.

b) Múltiples dispositivos implicados obliga a coordinación, que a menudo no es suficientemente cuidadosa.

c) El acceso a programas de rehabilitación es tardío y suele producirse en periodos avanzados de enfermedad (el cúmulo de 
pruebas a favor de la mayor eficacia de intervenciones tempranas impone un necesario cambio en esta perspectiva).

d) La intensidad de la atención ambulatoria a personas con TMG es insuficiente y apenas se proporciona atención domiciliaria.

e) Medicalización inadecuada de los problemas psicosociales.

f) Insuficiente participación de los interesados y escasa implicación comunitaria de los profesionales y los servicios.

g) Amplia variabilidad en práctica clínica, derivada sobre todo por hábitos y percepciones de los equipos, no tanto como por iniciativas distintas de los implicados,

h) Es necesario reconsiderar atención a excluidos, buscando mantener el principio de la equivalencia de cuidados.

En el terreno de los acuerdos concretos, la Estrategia diferencia objetivos (que los responsables sanitarios de cada CCAA se comprometen a cumplir)

Como complemento a los contendidos de esta tabla, el propio documento de la Estrategia define aspectos específicos referidos al plan integrado de atención (PIA) y a la red de

Tabla 1

Objetivos que se refierem a RPS la estrategia en materia de RPS

Aumentar el porcentaje de pacientes con trastornos mentales graves que son incluidos en un programa de rehabilitación

Aumentar el porcentaje de familias de pacientes con trastornos mentales graves que reciben un programa de intervención familiar específico, para mejorar su capacidad de afrontar las crisis y prevenir las recaídas

Proporcionar a los y las pacientes con trastorno mental grave un adecuado cuidado de su salud general

Las Comunidades Autónomas contemplarán, en sus planes de salud mental, las necesarias especificidades para la atención especializada de las personas con discapacidad intelectual y trastorno mental

El Ministerio de Sanidad y Consumo desarrollará, junto con las Comunidades Autónomas, un modelo de colaboración con los órganos de Justicia e Instituciones Penitenciarias para mejorar la atención de las personas con trastornos mentales sometidas al Código Penal a la Ley del Menor, asegurando la continuidad y la equivalencia de cuidados con el resto de la población

Establecer en cada una de las estructuras territoriales sanitarias el acceso a todos los dispositivos o programas terapéuticos, incluyendo los rehabilitadotes, suficientes para encarar las necesidades de su población, asegurando la continuidad asistencial a través de una red integrada de servicios en la que estarán incluidos los hospitales generales, y coordinados con atención primaria

Establecer, para las personas con trastornos mentales en tratamiento, un plan individualizado de atención Disponer en los equipos de salud mental en el ámbito comunitario, y en relación con los trastornos mentales graves prolongados, de un sistema organizativo para evitar los abandonos, facilitar la adherencia y que incluya la atención domiciliaria, así como la gestión y la coordinación multisectorial de su proceso assistencial

Otros objetivos también afectan indirectamente a la rehabilitación, al indicar que deben implantarse protocolos clínicos de los procesos de atención más prevalentes y/o de mayor complejidad asistencial teniendo em cuenta la perspectiva de género y la problemática de los colectivos de riesgo; e igualmente cuando se refiere a la implantación de guías integradas de práctica clínica.

Tabla 2

Recomendaciones sobre la rehabilitación en la Estrategia

Todos los y las pacientes diagnosticados de trastorno mental grave deben tener la posibilidad de ser incluídos precozmente en un programa de rehabilitación.

Se recomienda mejorar ale acceso a todos los dispositivos o programas terapéuticos y rehabilitadotes, tanto de la población adulta como infanto-juvenil, en las estructuras territoriales sanitarias. Estos dispositivos deben tener en cuenta las diferencias sociofamiliares de mujeres y hombres.

Se proponen dos tipos de planes de atención individualizada: el Plan Integrado de Atención para los pacientes con Trastorno Mental Grave, y el Acuerdo Terapéutico para los pacientes con Trastorno Mental Común.

Se recomiendan formas organizativas orientadas a mejorar la continuidad de la asistencia de las personas com trastorno mental grave, reducir el número de hospitalizaciones y mejorar su funcionamiento social y calidad de vida. Se proponen dos modelos de referencia: el "Tratamiento Asertivo Comunitario" y los "Programas de Continuidad de Cuidados".

Se recomienda la protocolización de los siguientes procesos de atención : Trastorno mental grave del adulto, trastorno mental grave de la edad avanzada, trastorno mental grave de la personalidade (e incluye recomendaciones de protocolización para otros procesos de atención).

recursos: Unidad/centro/servicio de salud mental comunitaria; Unidad de hospitalización en hospital general; Atención a urgencias 24 horas al día; interconsulta y enlace en los hospitales generales; Hospitalización de día para adultos; Programas comunitarios de rehabilitación (incluyendo programas de seguimiento comunitario y/o programas de seguimiento comunitario asertivo o similares); Rehabilitación en régimen de día; Rehabilitación con soporte residencial u hospitalario; Unidad 
de cuidados prolongados; Comunidad terapéutica para adultos; Alternativas residenciales con gradación de apoyo y actividad terapéutica o rehabilitadora; Programa de atención a domicilio; Hospitalización (atención intensiva) domiciliaria; Atención comunitaria 24 horas (incluyendo programas de seguimiento comunitario y/o programas de TAC o similares) y Programas de empleo con apoyo.

\section{Buenas prácticas}

El documento incluye una síntesis de "buenas prácticas" (programas bien definidos, y que han sido evaluados tras unos años de funcionamiento) que pueden servir de referencia para el desarrollo de nuevos dispositivos y programas ahí donde el despliegue de servicios es menor; varios de ellos se refieren a aspectos de la rehabilitación que se sintetizan en la tabla 3.

\section{Evaluación de la Estrategia}

Se efectuó una primera evaluación de la Estrategia tras los dos primeros años de su aprobación, cuyos resultados indican una tendencia por parte de las distintas Comunidades Autónomas a ampliar la implantación de los programas de RPS, si bien la mayor parte de los objetivos no podían considerarse sino

Tabla 3

Ejemplos de buenas prácticas en la Estrategia en salud mental del SNS que hacen referencia a la RPS ( sintesis) Intervención en familiares de pacientes con trastorno esquizofrénico: con el objetivo de Prevenir recaídas, mejorar funcionamiento social y capacidad de vida autónoma del Paciente, y el funcionamiento familiar. ( C. S. Ciudad Jardín .Alacant). Referencia : www.fundacionmaratotv3.org/simposium2006

Plan individualizado de rehabilitación. Trastornos mentales graves Desarrollado por los servicios especializados de RPS de Alcalá de Henares (Madrid). Referencia contacto: jgcases@grupoexter.com

Equipo de tratamiento asertivo comunitario de Avilés Atiende población de pacientes con TMG. Referencia contacto: juanjom@terra.es

Proceso asistencial integrado. Trastorno mental grave. Se desarrolla de acuerdo modelo de gestión por procesos en la Comunidad Andaluza. Referencia contacto : Rafael.pino.lopez.sspa@juntadeandalucia.es

parcialmente alcanzados. En el caso de el objetivo que señalaba la necesidad de que las Comunidades Autónomas establecieran un marco general que determine la implicación y el papel de la "Ley de Promoción de la Autonomía Personal y Atención a las personas en Situación de Dependencia"6, apenas se habían iniciado acciones en esa línea, siendo uno de los objetivos más importantes que queda pendiente de cumplir. Por otra parte, de acuerdo a la revisión de la bibliografía internacional efectuada sobre nuevos resultados de eficacia y eficiencia de intervenciones, se decidió ampliar o modificar algunos objetivos, tales como: 1) incluir la atención domiciliaria y la colaboración con atención primaria para atender las urgencias psiquiátricas y situaciones de crisis, 2) incluir también la atención domiciliaria entre los dispositivos y programas terapéuticos a los que debe garantizarse el acceso para la población afecta por problemas de salud mental 3 ) acciones para identificar e intervenir precozmente en las personas con psicosis, 4) revisar las desigualdades de género en el acceso a los servicios para eliminar esas desigualdades, 5) aumentar el número de personas con trastornos mentales que reciben el servicio de empleo con apoyo o empleo protegido.

La segunda evaluación se inició esta prevista para 2012, pero en 2010 se iniciaron ya algunos trabajos necesarios para ello, entre otros se constituyó un grupo de trabajo para estudiar el estado actual de la "transversalidad y continuidad en salud mental" que incluye valorar la articulación entre servicios sociales y sanitarios (los resultados estarán disponibles previsiblemente en el mes de octubre y podrán consultarse en la página web del Ministerio de sanidad) ${ }^{7}$.

\section{La rehabilitación laboral de los pacientes mentales en España}

Es una de las lagunas de la rehabilitación, el área donde de una forma más gráfica y tangible se manifiesta discriminación hacia la persona afecta por problemas de salud mental. El pobre panorama de la inclusión laboral de los pacientes mentales habla de los efectos sobre este colectivo de la sociedad mercantil, aún más agudizado en estos dos últimos años de "crisis" del sistema financiero-económico, y la dejación de las administraciones públicas a la hora de propiciar la inclusión social. El marco legal de referencia a este respecto está configurado por la Ley de Integración Social del Minusválido (LISMI) (1982) y por otras normativas en torno al llamado "empleo protegido". La LISMI define como "minusválido" desde el punto de vista legal a toda persona a quien la correspondiente comisión de valoración le aprecie una discapacidad superior al 33\%, y define también un marco normativo para la creación de Centros Especiales de Empelo (CEE) que sería un formato de "empresa social".

Empleo protegido. Conjunto de ayudas económicas que proporciona la administración del Estado a las empresas cuando contratan personas reconocidas como "minusválidas" (bonificación del 100\% en cuota a la Seguridad Social, es decir exención de cotización por ese trabajador, subvenciones a la contratación indefinida - entre 9 y 12.000 euros por cada contrato indefinido a "minusválido"- , reducción de la indemnización por despido, subvención del 5\% del salario mínimo interprofesional y ayudas para que la empresa cuente con “equipos de apoyo"), además de marcar la obligación de que toda empresa de más de 50 trabajadores debe reservar al menos $2 \%$ de sus puestos de trabajo para personas con "minusvalía".

Aunque no disponemos de una evaluación suficientemente amplia y precisa de la situación en toda España, de las evaluaciones y revisiones efectuadas a este respecto (Esteban \& Jordán de Urríes, 2006; López Álvarez, 2010) podemos resumir lo siguiente: a) De las personas afectas por discapacidad mental, están trabajando solamente el $7 \%$, siendo el colectivo con menos 
empelo entre todos los discapacitados; y b) Una característica diferencial de este colectivo es que tienen peor formación de base que el resto de discapacitados: el 65\% tienen estudios primarios (a menudo, sin completar estos estudios primarios), y solo el 5\% tienen estudios universitarios.

Hay distintos modelos y estrategias seguidas en España para la integración laboral de la persona con enfermedad mental: a) Como resultado de iniciativas privadas (asociaciones, fundaciones que promueven "empresa social, o centros especiales de empleo, de acuerdo a la legislación antes aludida), generalmente con algún tipo de apoyo financiero del sector público. Buenos ejemplos: La Fageda ${ }^{8}$ (una empresa agrícola, con fabricación industrial de productos lácteos), Egarintza ${ }^{9}$ (servicios de lavandería y otros), INTRAS ${ }^{10}$, o Isol ${ }^{11}$ (gestiona parque recreativo infantil y de adultos); b) Servicios con programas planificados desde los servicios públicos. Existen tres experiencias relevantes al respecto en España, en otras tantas Comunidades Autónomas, dos de ellas concebidas con criterios muy similares (FAISEM, en Andalucía y FISLEM, en Castilla La Mancha) como fundaciones públicas para promover la inserción social y sociolaboral de las personas con enfermedad mental y la que viene teniendo lugar en Madrid mediante la red de centros de rehabilitación laboral :

Modelo "fundaciones públicas". Ambas fundaciones, comparten el que surgen mediante acuerdos de gobierno de los Gobiernos Autonómicos respectivos, e implican acuerdos expresos de varios departamento, además del de Salud, tales como Trabajo y Empleo, Educación, Vivienda y Transportes. Promueven cuantas fórmulas pueden contribuir a mejorar la inclusión social, mediante el acceso a formación, promoviendo fórmulas de empleo con apoyo y de forma más específica, promoviendo la creación de centros especiales de empleoempresa social, donde las personas afectadas por TMG puedan trabajar, con su correspondiente contrato de trabajo.

Modelo CRL. El prototipo es la Comunidad de Madrid: dentro de la red de dispositivos sociales de apoyo a la rehabilitación en Madrid se ha ido desplegando una red de Centros de Rehabilitación Laboral (CRL). El CRL se define como un recurso específico que ofrece de un modo individualizado programas de rehabilitación laboral dirigidos a preparar y apoyar la inserción en el empleo de personas con problemas de salud mental, sobre todo en empresas ordinarias. El objetivo del CRL es ayudar a aquellos enfermos mentales que han alcanzado un buen nivel de autonomía a aprender o recuperar los hábitos y capacidades laborales necesarias para acceder al mundo laboral y apoyar su integración y mantenimiento en el mismo. Se ofrecen allí un abanico variado de actividades y programas tanto individuales como grupales: orientación vocacional, entrenamiento en hábitos básicos de trabajo, ajuste socio-laboral, talleres pre-laborales, apoyo a la formación laboral, búsqueda activa de empleo, apoyo y seguimiento de la inserción laboral. Cada CRL tiene una capacidad de 50 plazas (existen 18 en Madrid, lo que supone una ratio media de 22 plazas/100.000 habitantes) y cada centro cuenta con un psicólogo, un terapeuta ocupacional, un técnico de apoyo a la integración laboral, tres maestros de taller, además de un auxiliar administrativo y auxiliar de limpieza.

Empleo con apoyo ("primero coloca, después entrena"). Su implementación generalizada ha sido establecida como un objetivo de la Estrategia tras la evaluación, si bien aún no cuenta con mucha tradición en España ${ }^{12}$.

Las perspectivas de futuro de la RPS pueden sintetizarse en los siguientes aspectos ${ }^{13}$ :

a) Por parte de las administraciones:

- Garantizar disponibilidad y acceso a intervenciones que han demostrado su eficacia, tales como entrenamiento en habilidades sociales, psicoeducación individual y familiar, entrenamiento en el manejo de la enfermedad, tratamiento comunitario asertivo o gestión clínica intensiva de casos, psicoterapia de apoyo, o el empelo con apoyo.

- Revisar modelos organizativos de modo que se garantice red, coordinación, e integración; recordando que la rehabilitación no es sólo un conjunto de técnicas sino una estrategia de abordaje de los problemas de salud mental, sobre todo de la discapacidad y que debe orientarse, según las necesidades percibidas por los usuarios, en los servicios de salud mental y no confiarlo exclusivamente a los servicios muy especializados.

- Necesidad de considerar nuevos escenarios para la rehabilitación en contexto rural (Navarro, 2010), callesin hogar (como expone María Vázquez en otro artículo de esta publicación), prisión (Hernández Monsalve \& Herrera, 2003) ${ }^{14}$,

- Mantener RPS en la perspectiva de la salud pública. Generalizar acciones contra el estigma y la discriminación (Muñoz, Pérez Santos, Crespo, \& Guillén, 2006).

b) Por parte de administración y profesionales:

- Corregir errores: evitar que la circulación de los pacientes por los servicios debilite sus lazos y redes sociales (Bergero \& Gómez, 2000); evitar polarización de los servicios y programas.

- Rescatar antiguas prácticas (cuando no era tanto una cuestión técnica como un empeño de proyecto de ciudadanía - abrir espacios (Venturini, 2005): club sociales (participación, creatividad, además de socialización no estigmatizante), talleres de creatividad, expresión artística, y otras formas de ocio creativo (emisoras de radio, grupos musicales o de teatro).

- Por parte de profesionales, ampliar y mejorar sus técnicas:

a) Rescatar la dimensión subjetiva e intersubjetiva: integrar tratamiento y rehabilitación, trabajar en la perspectiva de la recuperación , abordar el duelo, considerar la resiliencia, trabajar sobre desempeño de roles (ejemplo: taller o escuela de padres), atender a la dinámica grupal y explorar nuevas posibilidades, como trabajar sobre el sentido del humor

b) RPS preventiva y promoción de la salud mental 
en población TMG. De acuerdo con buena parte de los usuarios de nuestros servicios, podremos suscribir la reivindicación de su palabra y su persona de acabar con la vieja consigna de todo para nosotros pero sin nosotros.

\section{Referencias}

Bergero, T., \& Gómez, M. (2000) El cuidador familiar en la psicosis: ¿es posible construir un lugar de encuentro entre la red sanitara formal e informal? In F. Rivas (Org.), La psicosis en la comunidad (pp. 307-332). Madrid. Asociación Española de Neuropsiquiatría.

Blasi Ras, R. (2000). La rehabilitación psicosocial integral en la comunidad y con la comunidad. In P. Escribá \& M. Huertas (Orgs.), Salud mental comunitaria: rehabilitación psico-social y reinserción socio-laboral. (pp. 309-330). Valencia Nau llibres.

Defensor del Pueblo (1992). Situación jurídica y asistencial del enfermo mental en España. Informes, estudios y documentos. Madrid: Publicaciones Defensor del Pueblo.

Desviat, M. (1994). La Reforma Psiquiátrica. Madrid: Ediciones Dor.

Escribá, P., \& Huertas, M. (2000). Salud mental comunitaria: rehabilitación psico-social y reinserción socio-laboral. Valencia: Nau llibres.

Espinosa, J. (1986). Cronicidad en psiquiatría. Madrid: Asociación Española de Neuropsiquiatría.

Esteban, R. (2002). La rehabilitación psicosocial en diferentes comunidades autónomas. In M. A. Verdugo, D. López, A. Gómez, \& M. Rodríguez, M. (Orgs), Rehabilitación en salud mental. Situación y perspectivas. (pp. 267272). Salamanca: Amarú.

Esteban, B., \& Jordán de Urríes, F. B. (2006). Empelo con apoyo para personas con discapacidad intelectual y para personas con enfermedad mental. Comparación metodológica de dos proyectos piloto. Siglo Cero, 37(2), 63-78.

European Communities, Health and Consumer Protection Directorate-General. (2005). European Comission Green Paper. Improving the mental health of the population: Towards a strategy on mental health for the European Union. COM 14/10/2005. Brussels 2005. Recuperado de http://ec.europe. eu/ph_deterinants/life_style/metal/green_paper/mental_grp-en.pdf

European Comission. Health and Consumer Protection Directorate-General (2008). European Pact for Mental Health and Wellbeing. Recuperado de http://ec.europe.eu/health/ph_determinants/life-style/mental/docs/ pact_en.pdf

Fernández, A. (2000). La familia como institución supletoria. La cara amarga de la Reforma Psiquiátrica. In: F. Rivas (Org.), La psicosis en la comunidad. (pp. 333-350). Madrid: Asociación Española de Neuropsiquiatría.

García, J., Espino, A., \& Lara, L. (1998). La Psiquiatría en la España de fin de siglo. Madrid: Editorial Díaz de Santos.

Gisbert, C. (2002). Rehabilitación psicosocial del trastorno mental severo. Situación actual y recomendaciones. Cuaderno Técnico $\mathrm{n}^{\circ} 6$. Madrid: Asociación Española de Neuropsiquiatría.

González de Chávez, M. (1980). La transformación de la asistencia psiquiátrica. Madrid: Mayoría.

Harding, C. M., \& McCrory, D. J. (2008). Relación entre los abordajes psicoterapéuticos y la rehabilitación psiquiátrica en pacientes con enfermedades mentales graves y persistentes. Una llamada hacia estrategias más integradoras. In: Y. Alanen, M. González de Chávez, A. L. Silver, \&
B. Martindale, B. (Orgs.), Abordajes psicoterapéuticos de las psicosis esquizofrénicas. (pp. 371-387) Madrid: Fundación para la investigación y el tratamiento de la esquizofrenia y otros trastornos.

Hernández Monsalve, M. (2000). Los programas de tratamiento comunitario asertivo: Una alternativa a la hospitalización ¿y algo más? In F. Rivas (Org.). La psicosis en la comunidad (pp. 131-189). Madrid: Asociación Española de Neuropsiquiatría.

Hernández Monsalve, M. (2002). La rehabilitación psicosocial en la encrucijada de la reforma psiquiátrica en España. La experiencia de Madrid. In M. A. Verdugo, D. López, A. Gómez, \& M. Rodríguez (Orgs.), Rehabilitación en salud mental. Situación y perspectivas. (pp. 311-326). Salamanca. Amarú.

Hernández Monsalve, M., \& Herrera, R. (2003). La atención a la salud mental de la población penitenciaria. Madrid: Asociación Española de Neuropsiquiatría.

Hernández Monsalve, M. (2010). Perspectivas de futuro. In A. Pastor, A. Blanco, \& D. Navarro (Orgs.), Manual de Rehabilitación (pp. 713-734). Madrid: Pirámide.

Ley $n$. 13 de 7 de abril de 1982. (1982, 7 de abril). Integración social de los minusválidos. Recuperado de http://sid.usal.es/idocs/F3/3-1161/lismi.PDF

Ley n. 14 de 25 de abril de 1986. Ley General de Sanidad. Boletín Oficial del Estado, 29/04/1986, pag 15.020 a 15.224 .

López Álvarez, M. (2010). El empleo y la recuperación de personas con trastornos mentales. La experiencia de Andalucía. Norte de salud mental, 8(36),11-23.

Ministerio de Sanidad y Consumo (1985). Informe de la Comisión para la Reforma Psiquiátrica. Madrid: Autor.

Ministerio de Sanidad y Consumo (2007). Estrategia en Salud Mental del Sistema Nacional de Salud. Madrid: Autor.

Muñoz, M., Pérez Santos, E., Crespo, M., \& Guillén, A. (2006) Propuestas especificas para la reducción del estigma social en las personas con enfermedad mental grave y crónica. Madrid: Consejería de Familia y Asuntos Sociales. Comunidad de Madrid.

Navarro D. (2010). Adaptación de la rehabilitación en contextos rurales. In A. Pastor, A. Blanco, A., \& D. Navarro (Orgs), Manual de Rehabilitación (pp. 713-734). Madrid: Pirámide.

Rebolledo, S. (1997). Rehabilitación Psiquiátrica. Santiago de Compostela: Universidad de Santiago de Compostela.

Rodríguez, A. (1997): Rehabilitación Psicosocial de personas con trastornos mentales crónicos. Madrid: Editorial Pirámide.

Saraceno, B., \& Montero, F. (1993). La rehabilitación entre modelos y prácticas. In V. Aparicio (Org.), Evaluación de Servicios en Salud Mental. (pp. 313321). Madrid: Asociación Española de Neuropsiquiatría.

Shephred, G. (1996). Avances recientes en rehabilitación. In J. A. Aldaz \& C. Vázquez (Orgs.), Esquizofrenia: fundamentos psicológicos y psiquiátricos de la rehabilitación (pp. 1-22). Madrid: Siglo XXI.

Uriarte, J. J. (2007). Psiquiatría y rehabilitación: la rehabilitación psicosocial en el contexto de la Enfermedad Mental Grave. Cuadernos de Psiquiatría Comunitaria, 7(2), 87-104.

Venturini, E. (2005). En realidad el verdadero problema no es cerrar sino abrir. El caso de Imola. Anais do I Congreso La salud mental es cosa de todos. El reto de la atención comunitaria de la persona con trastorno mental grave desde los servicios sociales (pp. 9-13). Madrid: IMSERSO.

World Health Organization (2005). Mental Health: Facing the Chalenges, Building Solutions. Report from the WHO European Ministerial Conference of Helsinki, Copenhagen. Recuperado de http://www.euro.who.int/ metalhealth2005 
1. Es una institución integrada por los consejeros de sanidad de todas las Comunidades Autónomas del Estado Español, junto al ministro de Sanidad del Gobierno.

2. La atención que se prestaba a quienes no tenían medios económicos, los "pobres", generalmente de muy baja calidad. 3. Ministerio de Sanidad y Consumo (2007). Estrategia en Salud Mental del Sistema Nacional de Salud. Madrid.

4. Asociación madrileña de familiares y amigos de enfermos con esquizofrenia.

5. Más Información disponible sobre pormenores de la evolución de la RPS en España en Rebolledo (1997); Rodríguez (1997); Escribá y Huertas (2000); Hernández Monsalve (2000; 2002).

6. Promulgada en 2006, se dirige a todo el colectivo de personas con discapacidad, incluyendo a quienes padecen TMG; su aplicación en la práctica choca con problemas administrativos y de financiación.

7. Recuperado de http://www.mspsi.es

8. Recuperado de http://www.fageda.com

9. Recuperado de http://www.egarintza.org/cas

10. Recuperado de http://www.intras.es

11. Recuperado de http://www.isolmurcia.org

12. Una nueva iniciativa al respecto es la Fundación SALTO que surgió con ese propósito: http://www.fundacion-salto.org

13. Según he expuesto más ampliamente en otra publicación reciente (Hernández Monsalve, 2010)

14. En los últimos años se está desarrollando un nuevo programa de rehabilitación psicosocial dirigido a las personas con TMG en prisión, que integra la actividad del personal sanitario y no sanitario (PAIEM ).

Mariano Hernández Monsalve, é psiquiatra, chefe dos serviços de saúde mental do Distrito de Tetuán, Madrid-Espanha. Endereço para correspondência: C/ Maudes, nº 32, Madrid (DP 28003). España. Tel.: ++34 91534 7363. E-mail: hergoico@gmail.com 